\title{
FRECKLED INSTANTONS IN TWO AND FOUR DIMENSIONS
}

\author{
A. Losev ${ }^{a}$, N. Nekrasov ${ }^{b}$, S. Shatashvili 曲 \\ ${ }^{a, b}$ Institute for Theoretical and Experimental Physics, Moscow, Russia \\ ${ }^{b}$ Physics Dept., Princeton University, Princeton NJ 08544 USA \\ ${ }^{c}$ Physics Dept., Yale University, New Haven CT 06540 USA
}

Field theory with instantons can be partially regularized by adding degrees of freedom at some scale. These extra degrees of freedom lead to the appearence of the new topological defects. These defects which we call freckles have some characteristic size depending on the scale at which the extra degrees of freedom revive.

The examples of two dimensional sigma model, four dimensional gauge theory are studied. The compactification of the four dimensional supersymmetric gauge theory down to two dimensions is also considered and the new phenomena are found.

Contribution to the Proceedings of Strings'99 Conference, Potsdam, July 1999

ITEP-TH-61/99

\section{Introduction}

Two dimensional sigma models and four dimensional gauge theories are similar in many ways[23]. The interesting aspect of the both theories is the presence of instantons, i.e. finite action solutions of the theory in the Euclidean space-time. Also, in both theories the conformal invariance of the instanton equations leads to the phenomenon of the instanton shrinking. In this way the instanton moduli space extends into the ultraviolet regions of the field space where the degrees of freedom of more fundamental theory may appear.

These degrees of freedom effectively compactify the instanton moduli space, by adding the smooth field configurations which look as singular point-

\footnotetext{
${ }^{1}$ On leave of absence from Steklov Mathematical Institute, St.-Petersburg, Russia
} 
like objects from the point of view of low-energy observer. Their contribution to the correlation functions is the phenomenon in the intersection theory known as "the contribution of the connected component" (sect. 9.1,10.4 in [0]). Its subtraction is an impact of the RG flow and is more or less contained in the paradigm of integrating out of the fast degrees of freedom [21, 27].

In this short note we discuss briefly the concrete examples of this phenomena with implications to the studies of supersymmetric gauge theories in four dimensions.

\section{Two dimensional instantons with freckles}

Non-linear sigma model. Consider the $\mathcal{N}=2$ supersymmetric sigma model (NLSM) with the target space $V=\mathcal{A} / / G$ - the Kähler quotient of the Kähler vector (affine) space $(\mathcal{A}, \omega)$ where $G$ acts preserving $\omega$. It has a moduli space $\mathcal{M}$ of BPS field configurations - instantons, represented by the holomorphic maps $\Phi$ of the worldsheet $\Sigma$ into $V$. This space $\mathcal{M}$ splits as a disjoint union of the spaces $\mathcal{M}_{\beta}$ labelled by $\beta=[\Phi(\Sigma)] \in \mathrm{H}_{2}(V, \mathbf{Z})$. The complex (virtual) dimension of $\mathcal{M}_{\beta}$ is given by:

$$
\operatorname{dim} \mathcal{M}_{\beta}=\int_{\beta} c_{1}(V)+\operatorname{dim} V(1-g)
$$

with $g$ being the genus of $\Sigma$.

Gauged linear sigma model. One can construct a gauged linear sigma model (GLSM), with chiral multiplets $\phi+\lambda+\ldots$ taking values in $A$, and vector multiplets $A+\psi+\ldots+\sigma$ taking values in $\operatorname{Lie} G$. The action of GLSM contains the potential term $\mathcal{V}=\langle\mu, \mu\rangle$ where $\mu: \mathcal{A} \rightarrow$ Lie $^{*} G$ is a moment map and $\langle$,$\rangle is a Killing form (D$-term).

At low energies the slowly varying field configurations are localized near the zeroes of $\mu$ and therefore describe the maps to $V=\mu^{-1}(0) / G$.

However, one may be interested in BPS field configurations, and these obey the following equations [27]:

$$
F_{A}=-e^{2} \mu, \quad \bar{\partial}_{A} \phi=0
$$

with $e$ being the gauge coupling.

Topological classification. The moduli space $\widetilde{\mathcal{M}}$ of BPS fields splits as a disjoint union of the spaces $\widetilde{\mathcal{M}}_{\delta}$ corresponding to the topologically distinct 
gauge field configurations. The latter are labelled by $\delta \in \mathrm{H}^{2}\left(\Sigma, \pi_{1}(G)\right) \approx$ $\mathrm{H}_{1}(G)$. In general there is no isomorphism between $\mathrm{H}_{1}(G)$ and $\mathrm{H}_{2}(V, \mathbf{Z})$. As a consequence, the BPS configurations in the GLSM and NLSM are labelled by different groups. However, the canonical principal $G$-bundle over $V$ gives an element (characteristic class) of the group $c \in \mathrm{H}^{2}\left(V, \mathrm{H}_{1}(G)\right)$ and we get a $\operatorname{map} \mid$ |f: $\mathrm{H}_{2}(V, \mathbf{Z}) \rightarrow \mathrm{H}_{1}(G):$

$$
\beta \mapsto \delta=\int_{\beta} c \in \mathrm{H}_{1}(G)
$$

Holomorphic data. The second equation in (2) tells us that $\phi$ is a holomorphic section of a vector bundle $\mathcal{E}$, whose holomorphic structure is specified by $\bar{\partial}_{A}$. The space of such sections is denoted by $\mathrm{H}^{0}(\mathcal{E})$ - it is a vector (affine, finite-dimensional) space for vector (affine, finite-dimensional) $\mathcal{A}$. One has Riemann-Roch formula:

$$
\operatorname{dim} H^{0}(\mathcal{E})-\operatorname{dim}^{1}(\mathcal{E})=d+\operatorname{rk} \mathcal{E}(1-g)
$$

where $d=\operatorname{deg} \mathcal{E}=\frac{1}{2 \pi i} \int_{\Sigma} \operatorname{Tr} F_{A}, \quad \operatorname{rk}(\mathcal{E})=\operatorname{dim} \mathcal{A}$. If there is a bound $\operatorname{Tr} i \mu \geq$ $-r$ then only for $d \leq 2 \pi e^{2} r$ that (21) have solutions (this is the sigma model analogue of the Donaldson jumps in four dimensional gauge theory).

The space $\mathcal{A}$ is acted upon the complexification $G_{c}$ of $G$. The potential $\mathcal{V}$ viewed as a function on $\mathcal{A}$ induces a gradient flow with respect to the Kähler metric on $\mathcal{A}$ along which the value of the potential decreases. The set $\mathcal{A}^{s}$ of points which flow to the zero set of $\mathcal{V}$ is distinguished for one has: $V=\mathcal{A}^{s} / G_{c}$. The set of critical points of $\mathcal{V}$ may have several connected components. For a coadjoint orbit $\lambda$ of $G$ denote by $C_{\lambda}$ the set of critical points $x$ of $\mathcal{V}$ such that $\mu(x) \in \lambda$, and by $S_{\lambda}$ the points $y$ which flow to $C_{\lambda}$ under the paths of the steepest descend of $\mathcal{V}$. The set of $\lambda$ for which $C_{\lambda}$ is not empty is denoted by $\Lambda$.

The spaces $\mathcal{H}=\mathrm{H}^{0}(\mathcal{E})$ and $\mathcal{H}^{\prime}=\mathrm{H}^{1}(\mathcal{E})$ form a pair of vector bundles (actually their difference is a better defined object as both may change dimension) over the moduli space $\mathbf{M}_{\Sigma}$ of holomorphic bundles on $\Sigma$. Actually, not every holomorphic bundle is good. More precisely, the equation $F_{A}=-e^{2} \mu$ picks out only stable pairs $\left(\bar{\partial}_{A}, \phi\right)$ [5]. The quotient of the space of stable pairs by the action of the complexified gauge group is the moduli

\footnotetext{
${ }^{2}$ It is the ordinary topological rather then algebraic homology of the group $G$ that we use throughout this paper
} 
space of the BPS configurations. It is simple to evaluate its dimension assuming that $\mathcal{H}^{\prime}=0$. Since the complex dimension of $\mathbf{M}_{\Sigma}$ equals $\operatorname{dim} G(g-1)$ and $\operatorname{dim} V=\operatorname{dim} \mathcal{A}-\operatorname{dim} G$ then:

$$
\operatorname{dim} \widetilde{\mathcal{M}}_{\delta}=\langle\delta\rangle+\operatorname{dim} V(1-g)
$$

If $\delta=\int_{\beta} c$ for $\beta \in \mathrm{H}_{2}(V, \mathbf{Z})$ then $\langle\delta\rangle=\int_{\beta} c_{1}(V)$.

From now on put $g=0$. In this case $\mathbf{M}_{\Sigma}$ is empty and the holomorphic bundles have a stabilizer isomorphic to $G_{c}$. The space $\mathcal{H}$ is acted upon by the group $G_{c}$. The subset of $\mathcal{H}$ which consists of the points whose $G_{c}$ orbit intersects the space of solutions to the equation $F_{A}=-e^{2} \mu$ is denoted by $\mathcal{H}^{s}$. The space of BPS field configurations is therefore $\widetilde{\mathcal{M}}=\mathcal{H}^{s} / G_{c}$. The moduli space $\widetilde{\mathcal{M}}$ contains a subset $\mathcal{M}$ of gauge equivalence classes of sections whose value at each point of $\Sigma$ belongs to $\mathcal{A}^{s}$. The $\phi$ 's from the complement $\widetilde{\mathcal{M}} \backslash \mathcal{M}$ violate this condition at some points $z_{1}, \ldots, z_{k} \in \Sigma$, where they take values in $S_{\lambda_{1}}, \ldots, S_{\lambda_{k}}$.

Remark. There is an interesting interplay between the $G$-equivariant cohomology of $\mathcal{A}$, cohomology of $V$, the set $\Lambda$, and the stratification of $\widetilde{\mathcal{M}}$ [11]. $\widetilde{\mathcal{M}}$ as (partial) compactification of $\mathcal{M}$ was studied in [6, 9, 18, 27, 28]. It differs from the compactification by stable maps [10].

The points of $\widetilde{\mathcal{M}}$ are called freckled instantons, $z_{1}, \ldots, z_{k}$ are the freckles of the types $\lambda_{1}, \ldots, \lambda_{k}$ respectively. In physics literature they appeared as vortices [1, 27].

The size of the freckles. As a smooth solution to (2) the freckles of the type $\lambda$ have a size $\ell_{\lambda} \sim \frac{1}{e \sqrt{\langle\lambda, \lambda\rangle}}$ where we used the Killing form on $\operatorname{Lie}^{*} G$.

Compactness of $\widetilde{\mathcal{M}}$. If $\Lambda$ is finite then the number of extra topological defects one has added to the sigma model instantons is finite and their size is bounded from below. One expects $\widetilde{\mathcal{M}}$ to be compact in this case (of course assuming the compactness of $V$ ). However, if $\Lambda$ is infinite the space $\widetilde{\mathcal{M}}$ is non-compact. Correlation functions. In NLSM with the target space $V$ an interesting set of correlation functions is obtained as follows: for $C \subset V$ and $z \in \Sigma$ define the subsets $\mathcal{M}_{C}^{(2)}, \mathcal{M}_{C}^{(0)}(z) \subset \mathcal{M}$ as follows: $\mathcal{M}_{C}^{(2)}$ consists of such holomorphic maps $\Phi: \Sigma \rightarrow V$ that $\Phi(\Sigma)$ intersects $C ; \mathcal{M}_{C}^{(0)}(z)$ consists of holomorphic maps $\Phi$ such that $\Phi(z) \in C$. Take the submanifolds $C_{1}, \ldots, C_{k} \subset V$, let $\omega_{1}, \ldots, \omega_{k}$ be the Poincare duals (delta functions supported at) to $C_{1}, \ldots, C_{k}$

\footnotetext{
${ }^{3}$ These subsets may have self-intersections and cusps
} 
homology classes. Let $0 \leq p \leq k$. Then the correlations functions of the observables $\mathcal{O}_{\omega_{1}}^{(0)}\left(z_{1}\right), \ldots, \overline{\mathcal{O}}_{\omega_{p}}^{(0)}\left(z_{p}\right), \int \mathcal{O}_{\omega_{p+1}}^{(2)}, \ldots \int \mathcal{O}_{\omega_{k}}^{(2)}$ in the sigma model (see [25] for their definition) is equal to the intersection number:

$$
\# \mathcal{M}_{C_{1}}^{(0)}\left(z_{1}\right) \cap \mathcal{M}_{C_{p}}^{(0)}\left(z_{p}\right) \cap \mathcal{M}_{C_{p+1}}^{(2)} \ldots \cap \mathcal{M}_{C_{k}}^{(2)}
$$

In GLSM the corresponding correlation functions count the intersection numbers of the closures $\widetilde{\mathcal{M}}_{C}^{(0)}(z), \widetilde{\mathcal{M}}_{C}^{(2)}$ of the submanifolds $\mathcal{M}_{C}^{(0)}(z), \mathcal{M}_{C}^{(2)}$ in $\widetilde{\mathcal{M}}$. In general they differ considerably as the submanifolds $\widetilde{\mathcal{M}}_{C}$ tend to intersect over the set of instantons with freckles [14.

So, are the freckles a nuisance or can they be helpful? One answer to this question is suggested in [14 where it is shown that in principle one could subtract the contribution of the freckles to get the genuine answers from NLSM. Or, in certain correlation functions the freckles are harmless. For example, if $c_{1}(V)>0$ then freckles do not contribute to the quantum cohomology ring.

Finally, as we shall see in the next section, the presence of freckles can be necessary in order to build up a higher-dimensional theory.

\section{Four dimensional theory on a product of two Riemann surfaces}

Take a genus $h$ Riemann surface $C$, a compact semi-simple Lie group $H$ and consider the moduli space of flat $H$-connections on $C$. To make things smooth we assume that $H$ has a center $Z$ and consider the flat connections in $H / Z$ bundle $E$ with non-trivial $c_{1}(E) \in \mathrm{H}_{2}\left(C, \pi_{1}(H / Z)\right.$ ) ('t Hooft magnetic flux). This moduli space $V$ is an infinite-dimensional symplectic quotient of the space $\mathcal{A}$ of all $H$-gauge fields (we omit the details concerning $Z$ here for brevity, they can be found in [26]) by the action of the infinite-dimensional group $G$ of $H$-gauge transformations. The space $V$ has a canonical generator of $\mathrm{H}^{2}(V)$ - the curvature of the determinant line bundle. We may take as the Kähler form on $V$ the $k$-th multiple of this generator. The number $k$ is called the level.

Four dimensional gauge theory emerges if we were to study the corresponding GLSM [3]. Indeed the matter fields $\Phi$ are the gauge fields on $C$ which also vary along the worldsheet $\Sigma$, the $G$ gauge fields are simply the gauge fields 
on $\Sigma$ which also vary along $C$ so altogether we get an $H$ gauge field on $\Sigma \times C$. The rest of the supermultiplets makes up a four dimensional partially twisted $\mathcal{N}=2$ vector multiplet.

Freckles. Having learned the lessons of the previous section we immediately raise the question of the interpretation of the freckles. The BPS configurations in the GLSM turn out to be precisely four dimensional instantons on $\Sigma \times C$. The two dimensional gauge coupling $e^{2}$ is inversely proportional to the area of $C, e^{2} \sim \frac{1}{k \text { Area } C_{C}}$, while the four dimensional coupling is related to the level $k$ as $g^{2} \sim \frac{1}{k}$. The labels $\lambda$ are interesting. They are nothing but the dominant weights of $H$ and correspond to the higher critical points of the Yang-Mills functional on $C$. The size of the type $\lambda$ freckle is therefore:

$$
\ell_{\lambda}^{2} \sim \frac{k \text { Area }_{C}}{\langle\lambda, \lambda\rangle}
$$

Compactness of the instanton moduli space. The fact that one can have the freckles of the arbitrarily small size is related to the four dimensional phenomenon of the instanton shrinking. One way to cure this problem is to replace the instantons by the torsion free sheaves, as in [2, 8, 22, 12, 19], or the non-commutative instantons [20]. $\Sigma \times C$ being Kähler allows that. There are new phenomena which one encounters after this compactification is performed. To be specific we study $U(2)$ case. The classical instanton moduli space splits as a $U(1)$ moduli space of flat connections on $\Sigma \times C$ and an $S O(3)$ moduli space. Moreover the parity of the $c_{1}$ of the $U(2)$ bundle is correlated with $w_{2}$ of the $S O(3)$ bundle, while $U(1)$ bundle can have an abritrary $c_{1}$ allowed by the instanton equations.

What happens when we hit a $4 \mathrm{~d}$ point-like instanton? If we are to treat this $4 \mathrm{~d}$ freckle in the holomorphic way we should replace a (stable) holomorphic rank two bundle $\mathcal{E}$ over the product of two curves $\Sigma \times C$ by the torsion free sheaf $\mathcal{F}$ which at some point ceases to be locally free. The space of such sheaves (for fixed underlying holomorphic bundle $\mathcal{E}^{\circ}$ and fixed position $z \times p$ of the freckle) is isomorphic to a $\mathbf{P}^{1}$ whose rôle is the following. Take a holomorphic bundle $\mathcal{E}^{\circ}$ of the instanton charge less then that of $\mathcal{F}$ by one. Fix a complex line $l$ in the fiber $\mathcal{E}_{z \times p}^{\circ}$ over a point $z \times p$. The space of such lines is a copy of $\mathbf{P}^{1}$ which we mentioned. Then the sheaf $\mathcal{F}_{l}$ is a sheaf of sections of $\mathcal{E}^{\circ}$ whose value at the point $z \times p$ belongs to $l$. One can show that the instanton charge of $\mathcal{F}$ is a sum: $\operatorname{ch}_{2}(\mathcal{F})=\operatorname{ch}_{2}\left(\mathcal{E}^{\circ}\right)-1$, (see [14 for details on the sheaves and freckles). 
Now let us look at what happens from the point of view of the two dimensional sigma model. We should restrict our sheaf onto each fiber $w \times C$, $w \in \Sigma$. If the restriction $\mathcal{F}_{w}$ is stable then the point $w$ is mapped to a point in the moduli space of stable rank two bundles on $C$, i.e. for such a point $w$ we are within the NLSM regime. If for some point $w \neq z$ we get an unstable bundle $\mathcal{F}_{w}$ then we get a two-dimensional freckle - the freckle of the GLSM. The type $\lambda$ of freckle is presicely the type of unstable bundle we got.

Finally, if we hit the point $w=z$ then the sheaf we get on $z \times C$ is a sheaf of sections of a vector bundle $E$ with smaller $c_{1}(E)$ then the first Chern class of the bundles over nearby fibers!

So the message here is that the four dimensional compactification adds in addition to the freckles which have to do with the unstable bundles of the same topology as the generic stable ones the bundles of different topology as well. We hope to return to more detailed investigation of this structure in future.

Quantum cohomology ring. Suppose that $\Sigma \approx \mathbf{S}^{2}$ and take the limit where Area $_{C} \ll$ Area $_{\mathbf{S}^{2}}$. We said above the quantum cohomology ring is not affected by freckles, so we may compute the quantum cohomology of $V$ using four dimensional gauge theory. The manifold $\Sigma \times \mathbf{S}^{2}$ has $b_{2}^{+}=1$ which is the situation where the Donaldson invariants get a contribution of the $u$-plane integral [16] in addition to a Seiberg-Witten contribution. It can be computed using the contact terms from [13] via the jumping techniques [16, 17]. The result is interesting to compare with the results of the computations in another chamber where $C$ is large and $\mathbf{S}^{2}$ is small in which case one finds [13] a Landau-Ginzburg type topological sigma model with the target space being a certain cover of the Seiberg-Witten $u$-plane. In particular the topological ring is infinite-dimensional in this case. To the contrary, the ring collapses once one passes through all the jumping walls. We present the answer for the $S O(3)$ case with $\left(w_{2},[C]\right) \neq 0$. The classical cohomology ring of $V$ is generated by the observables in the two dimensional Yang-Mills theory [26]:

$$
\begin{aligned}
& a=\int_{C} \mathcal{O}_{\operatorname{Tr} \phi^{2}}^{(2)}, \quad b=\mathcal{O}_{\operatorname{Tr} \phi^{2}}^{((0)} \\
& c=\sum_{i=1}^{h} \int_{A_{i}} \mathcal{O}_{\operatorname{Tr} \phi^{2}}^{(1)} \int_{B^{i}} \mathcal{O}_{\operatorname{Tr} \phi^{2}}^{(1)}
\end{aligned}
$$

Then the answer stemming from the four dimensional twisted $\mathcal{N}=2$ theory 
is:

$$
\begin{gathered}
\left\langle e^{\varepsilon_{1} a+\varepsilon_{2} b+\varepsilon_{3} c}\right\rangle \propto \\
\oint \frac{d u d z}{\left(u^{2}-1\right)^{g} z^{g+1}} e^{2 \varepsilon_{2} u+\left(\varepsilon_{1} u+\varepsilon_{3}\left(u^{2}-1\right)\right) z} \frac{\sigma_{3}\left(\varepsilon_{1}+z\right)}{\sigma\left(\varepsilon_{1}\right) \sigma_{3}(z)}
\end{gathered}
$$

where $\sigma_{3}(z)=1+\frac{u}{24} z^{2}+\ldots, \sigma(z)=z+\ldots$ are the Weierstraß elliptic functions associated to the SW curve:

$$
y^{2}=4 x^{3}-\frac{x}{4}\left(\frac{u^{2}}{3}-\frac{1}{4}\right)-\frac{1}{48}\left(\frac{2 u^{3}}{9}-\frac{u}{4}\right)
$$

The fact that the transcedental functions present in the similar expression valid in the other chamber cancel leading to the finite dimensional quantum cohomology ring is a serious check of the assertions above. It confirms the conjecture of [4].

Refined Donaldson-Witten theory?. In [13] it was argued that the four dimensional gauge theory has much more interesting observables then those studied in the Donaldson theory. In fact, to each invariant polynomial $I(\phi)$ on the Lie algebra of the gauge group one associates a descend sequence of observables, the highest term being the 4-observable which may be used for deforming the action. On the other hand, using the fact that the moduli space of flat connections is a Fano variety one can compute its genus zero Gromov-Witten invariants simply from the WDVV equations using the reconstruction theory of [10]. But, as we have argued above, the four dimensional instantons contain in addition to the two dimensional instantons also two dimensional freckles of various types. So if we were to compactify the four dimensional instanton moduli space we would certainly get the space which looks very much different from the moduli space of stable maps into $V$ above. Hence the computation of Gromov-Witten invariants, although produces the interesting function of the right number of variables (if the curves $C$ of all genera are considered) still misses the mark.

Higher dimensions. In principle we can construct the six-dimensional theory [3] at least at the level of its BPS field configurations by taking a two dimensional Riemann surface and studying its holomorphic maps into a moduli space of instantons on a Kähler fourfold $X$. Then, upon adding the freckles which are connections obeying $F^{2,0}=0$ and at the same time reducible

\footnotetext{
${ }^{4}$ the similar results were obtained independently in [15], although in a less concise form
} 
stationary points of the Yang-Mill functional we get a six dimensional BPS

gauge field. We believe these gauge fields are relevant in the study of D1-D5 systems.

\section{Acknowledgements}

We thank H. Braden, A. Gorsky and A. Rosly for discussions. The research of A. L. is partly supported by RFFI under the grant 98-01-00328, that of N. N by R.H.Dicke Fellowship from Princeton University, partly by RFFI under grant 98-01-00327; A. L. and N. N. are partially supported by grant 96-15-96455 for scientific schools. Research of S. Sh.is supported by DOE grant DE-FG02-92ER40704, by NSF CAREER award, by OJI award from DOE and by Alfred P. Sloan foundation.

\section{References}

[1] A. A. Abrikosov, Sov. Phys. JETP, 32 (1957) 1442; H. B. Nielsen and P. Olesen, Nucl. Phys. B61 (1973) 45

[2] W. Barth, K. Hulek, "Monads and Moduli of Vector Bundles", Manuscripta Math., 1978 v. 25, p. 323-347

[3] L. Baulieu, A. Losev, N. Nekrasov, Nucl. Phys B522 (1998) 82-104

[4] M. Bershadsky, A. Johansen, V. Sadov, C. Vafa, Nucl. Phys. B448 (1995) 166

[5] S. Bradlow, J. Diff. Geom.33 (1991) 169-213;

[6] V. Drinfeld, unpublished

[7] W. Fulton, "Intersection Theory", Springer-Verlag 1984

[8] D. Gieseker, "On the Moduli of Vector Bundles on Algebraic Surfaces", Ann. of Math. 1977, v.106, p. 45-60

[9] A. Givental, alg-geom/9603021

[10] M. Kontsevich, Yu. Manin, CMP 164 (1994) 525 
[11] F. C. Kirwan, "Sur la cohomologie des espaces quotients", C. R. Acad. Sci., Paris

[12] A. Losev, G. Moore, N. Nekrasov, S. Shatashvili, "Four Dimensional Avatars of 2d RCFT", Nucl. Phys.(Proc. Suppl.) 46 (1996) 130-145

[13] A. Losev, N. Nekrasov and S. Shatashvili, "Issues in topological gauge theory", Nucl Phys. B534 (1998) 549

[14] A. Losev, N. Nekrasov and S. Shatashvili, "Freckled Instantons", to appear in Y. Golfand Memorial Volume, M. Shifman Eds.: World Scientific, Singapore

[15] C. Lozano, M. Mariño, hep-th/9907165

[16] G. Moore, E. Witten, Adv. Theor. Math. Phys., 1 (1998) 298

[17] G. Moore, M. Mariño, hep-th/9804104

[18] D. Morrison, R. Plesser, Nucl. Phys. B440 (1995) 279-354

[19] H. Nakajima, "Lectures on Hilbert Schemes of Points on Surfaces", H. Nakajima's homepage

[20] N. Nekrasov, A. S. Schwarz, hep-th/9802068, CMP 198 (1998) 689

[21] V. Novikov, M. Shifman, A. Vainshtein, V. Zakharov, Nucl. Phys. B229 (1983) 381, 407

[22] K. Okonek, M. Schneider, H. Spindler, "Vector Bundles on Complex Projective Spaces", Birkhäuser Boston 1980

[23] A. Polyakov, Phys. Lett.B59 (1975) 79-81

[24] E. Witten, Nucl. Phys. B340 (1990) 281-332; R. Dijkgraaf, E. Witten, Nucl. Phys. B342 (1990) 486-522; R.Dijkgraaf, E. Verlinde, H. Verlinde, Nucl. Phys. B352 (1991) 59

[25] E. Witten, CMP 117 (1988) 353; CMP 118 (1988) 411

[26] E. Witten, "Two dimensional gauge theories revisited", J. Geom. Phys.9 (1992) 303 
[27] E. Witten, "Phases of $\mathcal{N}=2$ theories in two dimensions", Nucl. Phys.B403 (1993) 159-222

[28] E. Witten, "Verlinde Algebra and Quantum Cohomology of Grassmanian", hep-th/9312104 